\title{
Instrumentation for modeling of discharge processes in ignition capacitive systems
}

\author{
F. A. Gizatullin ${ }^{1} \cdot$ R. M. Salikhov ${ }^{1}$ (I) N. V. Efimenko ${ }^{1}$ A. G. Karimova ${ }^{1} \cdot$ A. U. Demin ${ }^{1}$
}

Received: 29 May 2018 / Accepted: 16 July 2019 / Published online: 24 July 2019

(c) The Author(s) 2019

\begin{abstract}
In this article, developing a computer model allowing to obtain analytically the dependences of the discharge current $i(t)$ and voltage in a spark discharge $U(t)$ on the given discharge circuit parameters is carried out. A toolkit for evaluating the characteristics of capacitive ignition systems of various types at the stages of creating new circuit technical solutions and engineering has been developed. The obtained results allow to significantly reduce the time for evaluating the potential of ignition systems without carrying out sufficiently complex and labor-intensive experimental studies. The resulting dependences $i(t)$ and $U(t)$ allow to determine and calculate all the main parameters of spark discharges in plugs-energy, power and duration of discharges, as well as the maximum value of the discharge current.
\end{abstract}

Keywords Ignition capacitive systems $\cdot$ Discharge processes $\cdot$ Current-voltage characteristics $\cdot$ Approximation

\section{Introduction: formulation of the problem}

The discharge circuits of capacitive ignition systems of all main types contain nonlinear elements-switching spark gaps and semiconductor or spark plugs. The nonlinearity of discharge circuits complicates the development of mathematical and computer models of discharge processes [1-4].

In [3], based on the results of experimental studies of discharge processes in a capacitive ignition system, the current-voltage characteristics of spark discharges in semiconductor plugs were constructed and approximated with respect to the oscillatory and unipolar types of discharge processes in the range of the parameters of the discharge circuits corresponding to serial and prospective capacitive ignition systems. The obtained expressions for the volt-ampere characteristics of the oscillatory and unipolar discharges are the basis for the development of tools for modeling and research of the efficiency of ignition systems at the stages of development of new circuit solutions and analysis of the efficiency of the projected ignition systems.

The article solves the problem of developing a computer model allowing to obtain analytically the dependences of the

\section{R. M. Salikhov}

texprom@yandex.ru

1 Department of Electromechanics, Ufa State Aviation Technical University, Karl Marx str. 12, Ufa, Russia 450000 discharge current $i(t)$ and voltage in a spark discharge $U(t)$ on the given discharge circuit parameters: storage capacitor capacitance $C$, discharge circuit inductance $L$, active resistance $R$ and commuting discharger breakdown voltage $U_{0}$ taking into account the current-voltage characteristics of spark discharges in semiconductor sparks for the main types of capacitive discharge-oscillatory and unipolar. The task was solved without taking into account the impact on the spark discharges of pressure in the combustion devices, temperature, physicochemical properties of fuels and the composition of the fuel mixture, determined by the air-fuel ratio. The tasks of estimating the igniting ability of spark discharges based on the consideration of gas-dynamic and thermodynamic processes in the combustion chambers and starting igniters of gas turbine engines were solved, including, in [5-8].

The main difficulty in developing a computer model of discharge processes in capacitive ignition systems, taking into account the actual current-voltage characteristics of spark discharges, is as follows: The discharge current in capacitive ignition systems varies according to the damped sinusoid law; therefore, for the entire discharge process, there is a family of current-voltage characteristics, each of which corresponds to the half-period of the current change.

As is known, the discharge in semiconductor plugs consists of two stages-preparatory and spark. During the preparatory stage, the current flows through the semiconductor 
element of the spark plug; due to the negative semiconductor resistance coefficient, an avalanche-like current and temperature rise to a state where explosive sparking of the semiconductor material and gas ionization between the electrodes over the semiconductor occur. This circumstance leads to the breakdown of the ionized gap above the semiconductor and the transition of the discharge to the spark stage, during which ignition of the combustible fuel mixture occurs. In the course of developing a computer model of discharge processes in capacitive ignition systems of an oscillatory and aperiodic discharge, the preparatory stage of the discharge was not taken into account, as it does not directly affect the processes in ignition. An additional justification for this is the fact that in high-voltage capacitive ignition systems with pulse transformers, the preparatory stage of discharges is practically absent $[4,9]$.

\section{The results of the development of a computer model}

The developed computer model of discharge processes implemented in Simulink, in accordance with the technology described in [10], is shown in Fig. 1. The model describes the discharge processes in the basic circuit of the discharge circuits of the capacitive ignition systems shown in Fig. 2.

When the key $\mathrm{S}$ is open, the discharge current through the spark plug $F$ after the breakdown of the spark gap FV varies

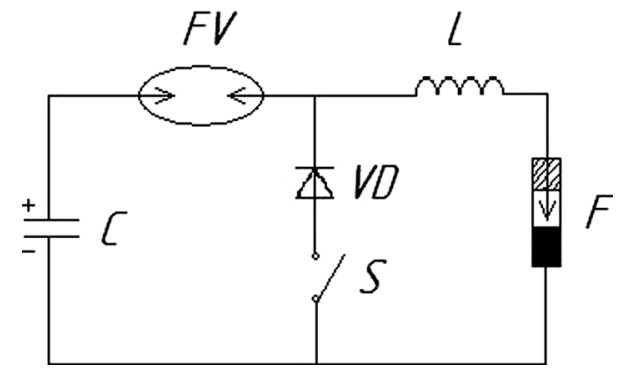

Fig. 2 Basic scheme of discharge circuits of capacitive ignition systems of oscillatory and unipolar discharge

according to a damped oscillatory law; with a closed key S, the current through the plug increases to a maximum value and then decreases unipolarly [3, 4].

The magnitude of the discharge current at the given fixed time is calculated in the MATLAB function block $(i)$, which generates the output signal $i$ using the input parameters $L, C$, $U_{0}$ and $R$, based on the functional dependence [4]:

$i=\frac{U_{0}}{\omega L} \cdot e^{-\delta t} \cdot \sin \omega t$

where $\delta=\frac{R}{2 L}$ is the damping coefficient; $\omega=\sqrt{\omega_{0}^{2}-\delta^{2}}$; $\omega_{0}=\frac{1}{\sqrt{L C}} ; U_{0}$ is the capacitor charge voltage $C ; L$ is the inductance of the discharge circuit; $R$ is the equivalent active resistance of the discharge circuit, including the resistance
Fig. 1 Computer model of discharge processes in the capacitive ignition system

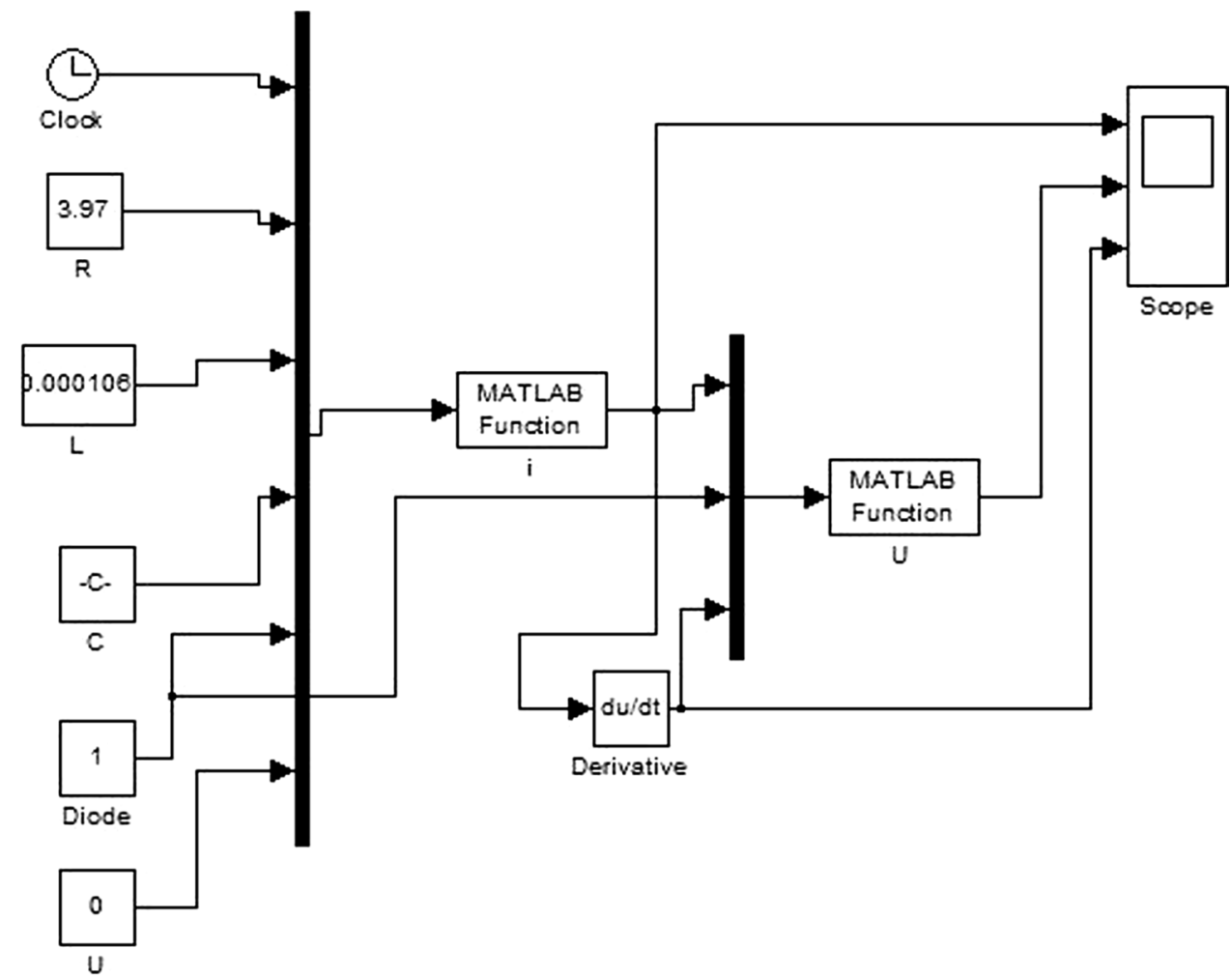


of the punctured spark gap FV and the spark plug F, the resistance of the inductance $L$ and the connecting wires.

The volt-ampere characteristics of the spark discharges of the oscillatory and unipolar types, containing the direct and inverse branches, were approximated in [3] by the corresponding mathematical dependencies. The direct branches of current-voltage characteristics of oscillatingtype discharges, as follows from the theory of a pulsed electric arc [11], correspond to an increase in current during each half-period of current change, while the opposite branches correspond to a decrease in current during each half-period. The forward branch of the current-voltage characteristic of unipolar-type discharges corresponds to an increase in current; the reverse branch corresponds to a decrease in current from the maximum value to zero.

For the forward and reverse branches of the current-voltage characteristics of spark discharges, the expressions are used [3]:

- for the forward branch $U_{1}(i)$ and the reverse branch $U_{2}(i)$ of the oscillation discharge:

$$
\begin{aligned}
& U_{1}(i)=a_{1}-b_{1} \cdot i+c_{1} \cdot i^{1,5}+\frac{d_{1}}{i^{0,5}} \\
& U_{2}(i)=a_{2}-b_{2} \cdot i+c_{2} \cdot i^{1,5}+d_{2} \cdot i^{0,5}
\end{aligned}
$$

- for the forward branch $U_{3}(i)$ and the reverse branch $U_{4}(i)$ of the current-voltage characteristic of the unipolar discharge:

$$
\begin{aligned}
& U_{3}(i)=a_{3}-b_{3} \cdot i+c_{3} \cdot i^{1,5}+\frac{d_{3}}{i^{0,5}} \\
& U_{4}(i)=a_{4}+b_{4} \cdot i^{1,5}-c_{4} \cdot i^{2}+d_{4} \cdot i^{3}
\end{aligned}
$$

The values of the coefficients $a_{1}, a_{2}, a_{3}, a_{4}, b_{1}, b_{2}, b_{3}$, $b_{4}, c_{1}, c_{2}, c_{3}, c_{4}, d_{1}, d_{2}, d_{3}, d_{4}$, which ensure a high correspondence of the approximating expressions (2)-(5) to real experimental current-voltage characteristics, in [3] are:

$$
\begin{aligned}
& a_{1}=65, b_{1}=0.017, c_{1}=0.00038, d_{1}=459.8 \\
& a_{2}=19,61, b_{2}=0.15, c_{3}=0.0014, d_{3}=4.904 \\
& a_{3}=65, b_{3}=0.017, c_{3}=0.00038, d_{3}=459.8 \\
& a_{4}=14,703, b_{4}=0.0015, c_{4}=3.26, d_{4}=1.9
\end{aligned}
$$

The dependencies for the voltage in the spark discharge $U(t)$ shown in Fig. 1 are calculated by the MATLAB function block $(U)$ according to the algorithm: At a positive current variation rate calculated by the derivative block, the dependences (2) or (4) are used; at a negative current variation rate, dependences (3) or (5) are used.

The oscillatory or unipolar type of the discharge in the computer model is set by the diode block.

\section{Confirmation of simulation results}

The adequacy of the developed model was estimated by comparing the obtained dependences $i(t), U(t)$ with the experimental dependences for the same values of the discharge circuit parameters $C, L, R$ and $U_{0}$ for oscillatory and unipolar types.

The scheme of the experimental bench is presented in Fig. 3.

Experimental studies were carried out in laboratory conditions without affecting the serial semiconductor plug of high pressure, temperature and fuel mixture. The size of the electrode gap of the spark plug was $0.6 \mathrm{~mm}$.

The effect of the conditions in the combustion chamber of a gas turbine engine on the duration of spark discharges and the maximum value of current through a semiconductor plug was investigated in [12].

The capacity of the storage capacitor was $C=0.5 ; 1.0$; $2.0 \mu \mathrm{F}$, inductance $\mathrm{L}=32,106 \mu \mathrm{H}$. As a switching element, a two-electrode spark gap R-44 with a breakdown voltage of $U_{0}=3.1 \mathrm{kV}$ was used. The charge of the storage capacitor was carried out from the regulated transformer power supply.

The repetition frequency of spark discharges in a semiconductor plug under the experimental conditions was

Fig. 3 Schematic diagram of the experimental stand

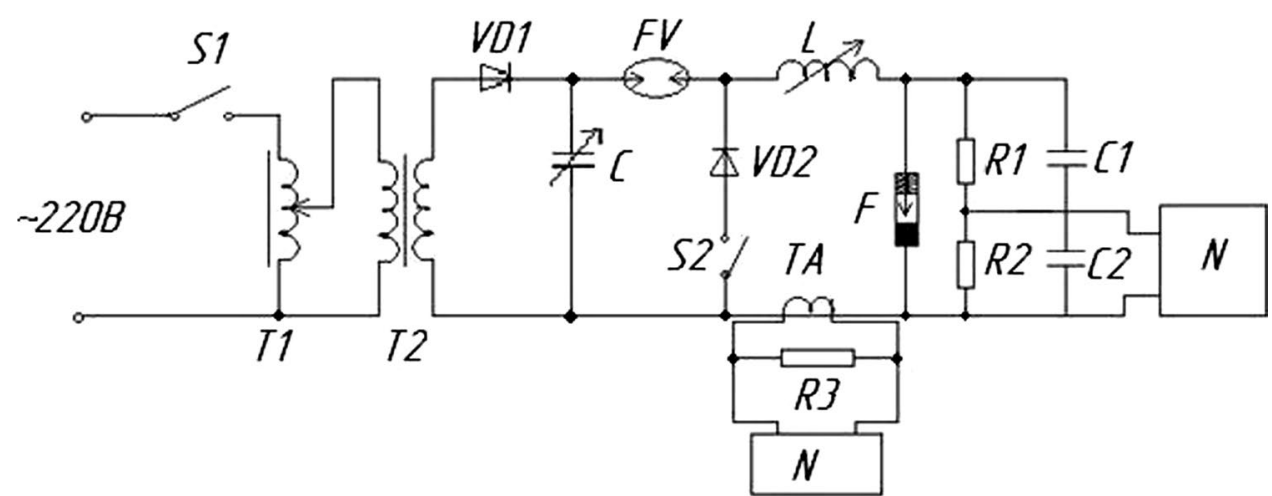


varied within 4-8 pulses per second; at the same time, the oscillograms of the discharge processes remained practically unchanged.

The oscillography of the discharge current was carried out using a current measuring transformer TA with a number of turns $w=100$ and a load resistor $R_{1}=0.8 \mathrm{O}$; oscillography of the voltage in a spark discharge in a plug was carried out using an ohmic voltage divider $R_{2}-R_{3}$ with a transmission factor of 100 .

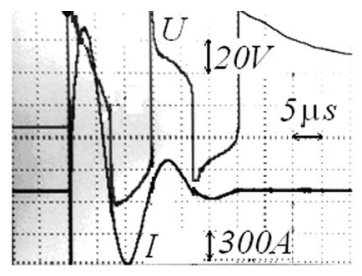

(a)

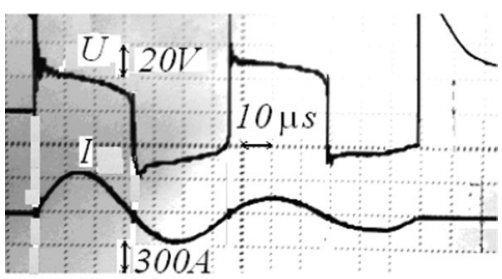

(b)

Fig. 4 Oscillograms for the oscillatory discharge. a $C=1 \mu \mathrm{F}$, $L=32 \mu \mathrm{H}, \mathbf{b} C=1 \mu \mathrm{F}, L=106 \mu \mathrm{H}$

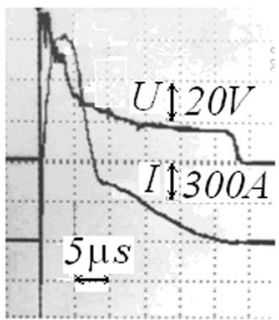

(a)

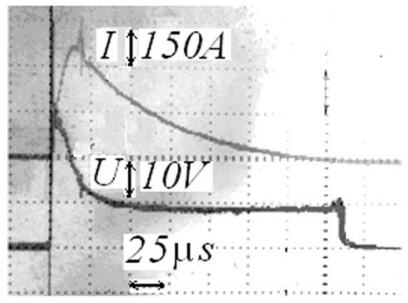

(b)

Fig. 5 Oscillograms of an unipolar discharge. a $C=1 \mu \mathrm{F}, L=32 \mu \mathrm{H}$, b $C=1 \mu \mathrm{F}, L=106 \mu \mathrm{H}$
Figures 4 and 5 show the characteristic oscillograms of the oscillatory and unipolar discharge obtained experimentally.

Figures 6 and 7 show the oscillograms $i(t)$ and $U(t)$ obtained using the computer model for the oscillatory and unipolar types of discharges with the same values of the storage capacitor capacitance and the discharge circuit inductance.

The energy efficiency of the storage capacitor, i.e., the ratio of the energy of spark discharges to the energy accumulated in the capacitor, under the experimental conditions was $15-20 \%$, and an increase in the inductance of the discharge circuit led to an increase in the energy utilization factor of the storage capacitor, which is consistent with the results of studies conducted in [4].
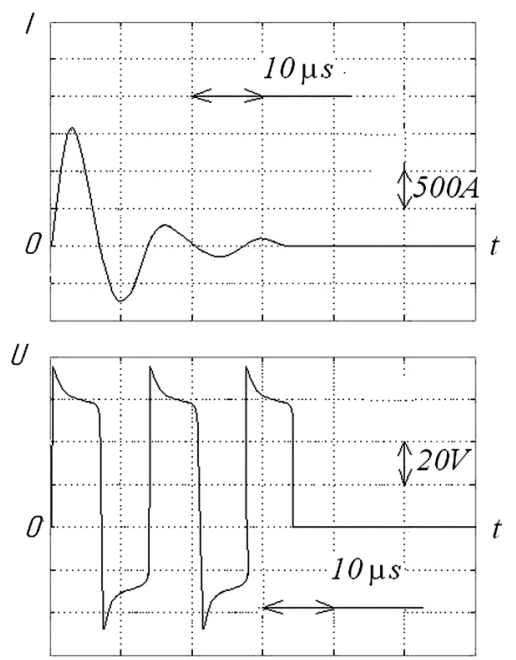

(a)
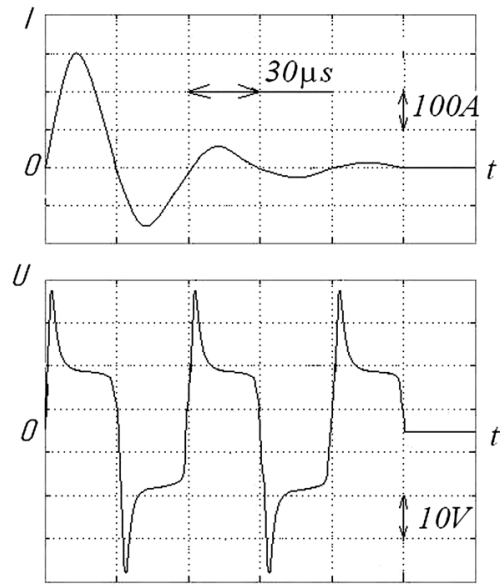

(b)

Fig. 6 Oscillograms of the discharge current and voltage drop in the spark plug for the oscillatory discharge. a $C=1 \mu \mathrm{F}, L=32 \mu \mathrm{H}$; b $C=1 \mu \mathrm{F}, L=106 \mu \mathrm{H}$ 

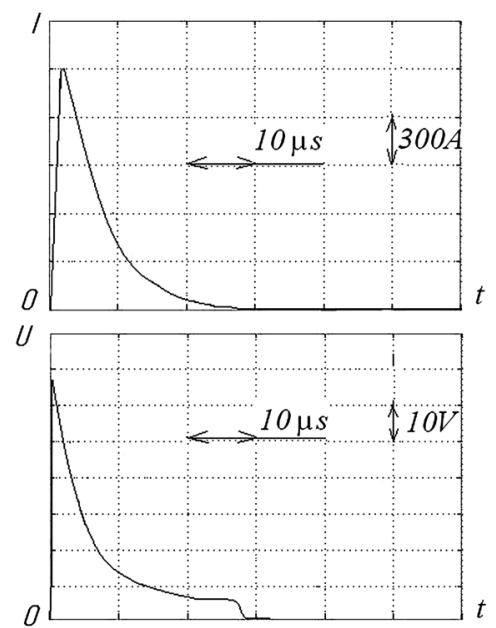

(a)
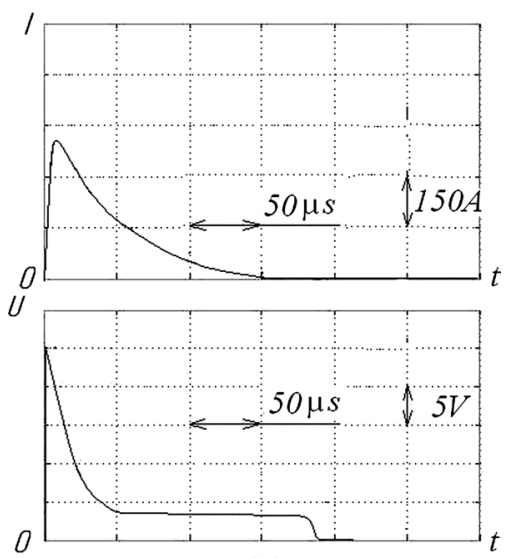

(b)

Fig. 7 Oscillograms of the discharge current and voltage drop in the spark plug for the unipolar discharge. a $C=1 \mu \mathrm{F}, L=32 \mu \mathrm{H} ; \mathbf{b}$ $C=1 \mu \mathrm{F}, L=106 \mu \mathrm{H}$

\section{Findings}

From Figs. 4, 5, 6 and 7, the discrepancy between the theoretical and experimental dependences $i(t)$ and $U(t)$ does not exceed $10 \%$, which is acceptable for engineering practice in relation to fast pulsed processes and indicates the adequacy of the developed computer model, taking into account the restrictions imposed by conditions in the zone of formation of spark discharges in semiconductor plugs.

The resulting simulations of $i(t)$ and $U(t)$ make it possible to determine and calculate all the main parameters of spark discharges in plugs - discharge current, energy, power, duration of discharges in laboratories and test benches without exposure to the fuel mixture, pressure and temperature.

Thus, tools have been developed for evaluating the characteristics of capacitive ignition systems of various types at the stages of creating new circuit solutions, research and design. The presented results will significantly reduce the time to assess the potential capabilities of ignition systems without conducting rather complex and time-consuming experimental studies in real combustion chambers of gas turbine engines.

Open Access This article is distributed under the terms of the Creative Commons Attribution 4.0 International License (http://creativeco mmons.org/licenses/by/4.0/), which permits unrestricted use, distribution, and reproduction in any medium, provided you give appropriate credit to the original author(s) and the source, provide a link to the Creative Commons license, and indicate if changes were made.

\section{References}

1. Gizatullin, F.A., Zinovev, K.V.: Simulation of discharge impulses of capacitive ignition system. Russ. Aeronaut. 2, 45-51 (2008)

2. Gizatullin, F.A., Salikhov, R.M., Ichsheikina, T.M.: Methods of measuring the energy of the spark discharges in the semiconductor plugs in the gas turbine engines. Aerosp. Instrum. Mak. 3, 10-14 (2013)

3. Gizatullin, F.A., Karimova, A.G.: Analysis of nonlinearities in semiconductor spark plugs. Electrotech. Complexes Syst. 4, 324327 (2015)

4. Gizatullin, F.A.: Capacitive Ignition Systems. UGATU, Ufa (2002)

5. Lefebvre, H., Ballal, D.R.: GAZ Turbine Combustion, 3rd edn. CRC Press, Boca Raton (2010)

6. Gizatullin, F.A.: On the theory of spark ignition of air-fuel mixtures. Aviat. Ind. 1, 56-60 (2000)

7. Xudong, Shi, et al.: Modeling and simulation of aviation engine ignition spark frequency disorder. Open Electr. Electron. Eng. J. 9, 193-199 (2015). https://doi.org/10.2174/1874129001509010193

8. Meng, X., et al.: Research on modeling and faults diagnosis of aero-engine ignition system. Open Autom. Control Syst. J. 6, 885-893 (2014). https://doi.org/10.2174/1874444301406010885

9. Mahajan, D.P., Narayanswamy, R., Saxena, S.K.: Novel exciter circuit for ignition of gas turbine engines in aerospace applications. In: 2013 IEEE International Conference on Industrial Technology (ICIT), Cape Town, pp. 613-617 (2013). https://doi. org/10.1109/icit.2013.6505741

10. Nuruzzaman, M.: Modeling and Simulation: Introduction for Scientists and Engineers. AuthorHouse, Bloomington (2004)

11. Taev, I.S., Buhl, B.K., Gogello, A.G., et al.: Fundamentals of the theory of electrical apparatus: Proc. for universities in the specialty "Electrical apparatuses"; Ed. I.S. Taeva. - M.: Higher. wk., (1987). - 352 with: Il

12. Vakhitov, R.Sh., Gizatullin, F.A., Komissarov, G.V.: Discharge processes in the ignition system with a semiconductor plug when starting a GTE. Aviat. Ind. 9, 24-25 (1979)

Publisher's Note Springer Nature remains neutral with regard to jurisdictional claims in published maps and institutional affiliations. 